\title{
Viscoelastic characteristics of the canine cranial cruciate ligament complex at slow strain rates
}

\author{
Rosti Readioff $^{\text {Corresp., } 1}$, Brendan Geraghty ${ }^{2}$, Ahmed Elsheikh ${ }^{1,3,4}$, Eithne Comerford ${ }^{\text {Corresp. } 2,5}$ \\ ${ }^{1}$ School of Engineering, University of Liverpool, Liverpool, United Kingdom \\ 2 Institute of Life Course and Medical Sciences, University of Liverpool, Liverpool, United Kingdom \\ 3 Beijing Advanced Innovation Center for Biomedical Engineering, Beihang University, Beijing, China \\ 4 UCL Institute of Ophthalmology, NIHR Moorfields BRC, London, United Kingdom \\ 5 School of Veterinary Science, University of Liverpool, Neston, United Kingdom \\ Corresponding Authors: Rosti Readioff, Eithne Comerford \\ Email address: r.readioff@leeds.ac.uk, eithne.comerford@liverpool.ac.uk
}

Ligaments including the cruciate ligaments support and transfer loads between bones applied to the knee joint organ. The functions of these ligaments can get compromised due to changes to their viscoelastic material properties. Currently there are discrepancies in the literature on the viscoelastic characteristics of knee ligaments which are thought to be due to tissue variability and different testing protocols.

The aim of this study was to characterise the viscoelastic properties of healthy cranial cruciate ligaments (CCLs), from the canine knee (stifle) joint, with a focus on the toe region of the stress-strain properties where any alterations in the extracellular matrix which would affect viscoelastic properties would be seen.

Six paired CCLs, from skeletally mature and disease-free Staffordshire bull terrier stifle joints were retrieved as a femur-CCL-tibia complex and mechanically tested under uniaxial cyclic loading up to $10 \mathrm{~N}$ at three strain rates, namely $0.1,1$ and $10 \% / \mathrm{min}$, to assess the viscoelastic property of strain rate dependency. The effect of strain history was also investigated by subjecting contralateral CCLs to an ascending $(0.1,1$ and $10 \% / \mathrm{min})$ or descending $(10,1$ and $0.1 \% / \mathrm{min})$ strain rate protocol.

The differences between strain rates were not statistically significant. However, hysteresis and recovery of ligament lengths showed some dependency on strain rate. Only hysteresis was affected by the test protocol and lower strain rates resulted in higher hysteresis and lower recovery. These findings could be explained by the slow process of uncrimping of collagen fibres and the contribution of proteoglycans in the ligament extracellular matrix to intra-fibrillar gliding, which results in more tissue elongations and higher energy dissipation.

This study further expands our understanding of canine CCL behaviour, providing data for material models of femur-CCL-tibia complexes, and demonstrating the challenges for engineering complex biomaterials such as knee joint ligaments. 


\section{Viscoelastic characteristics of the canine cranial cruciate 2 ligament complex at slow strain rates}

3

4 Rosti Readioff ${ }^{1}$, Brendan Geraghty ${ }^{2}$, Ahmed Elsheikh 1,3,4 and Eithne Comerford ${ }^{2,5}$ 5

$6 \quad{ }^{1}$ School of Engineering, University of Liverpool, Liverpool, UK.

$7 \quad{ }^{2}$ Institute of Life Course and Medical Sciences, University of Liverpool, Liverpool, UK.

$8 \quad{ }^{3}$ Beijing Advanced Innovation Center for Biomedical Engineering, Beihang University, Beijing, 9 China.

$10{ }^{4}$ UCL Institute of Ophthalmology, NIHR Moorfields BRC, London, UK

$11{ }^{5}$ School of Veterinary Science, University of Liverpool, Neston, UK.

12

13 Corresponding Authors:

14 Rosti Readioff ${ }^{1}$

15 Institute of Medical and Biological Engineering, School of Mechanical Engineering, University 16 of Leeds, Leeds, UK.

17 E-mail address: $\underline{\text { r.readioff@leeds.ac.uk }}$

18

19 Eithne Comerford 2,5

20 Institute of Life Course and Medical Sciences, University of Liverpool, Liverpool, UK.

21 School of Veterinary Science, University of Liverpool, Neston, UK.

22 E-mail address: Eithne.Comerford@liverpool.ac.uk 


\section{Abstract}

25 Ligaments including the cruciate ligaments support and transfer loads between bones applied to

26 the knee joint organ. The functions of these ligaments can get compromised due to changes to

27 their viscoelastic material properties. Currently there are discrepancies in the literature on the

28 viscoelastic characteristics of knee ligaments which are thought to be due to tissue variability and

29 different testing protocols.

30 The aim of this study was to characterise the viscoelastic properties of healthy cranial cruciate

31 ligaments (CCLs), from the canine knee (stifle) joint, with a focus on the toe region of the stress-

32 strain properties where any alterations in the extracellular matrix which would affect viscoelastic

33 properties would be seen.

34 Six paired CCLs, from skeletally mature and disease-free Staffordshire bull terrier stifle joints

35 were retrieved as a femur-CCL-tibia complex and mechanically tested under uniaxial cyclic

36 loading up to $10 \mathrm{~N}$ at three strain rates, namely $0.1,1$ and $10 \% / \mathrm{min}$, to assess the viscoelastic

37 property of strain rate dependency. The effect of strain history was also investigated by

38 subjecting contralateral CCLs to an ascending $(0.1,1$ and $10 \% / \mathrm{min})$ or descending $(10,1$ and

$390.1 \% / \mathrm{min})$ strain rate protocol.

40 The differences between strain rates were not statistically significant. However, hysteresis and

41 recovery of ligament lengths showed some dependency on strain rate. Only hysteresis was

42 affected by the test protocol and lower strain rates resulted in higher hysteresis and lower

43 recovery. These findings could be explained by the slow process of uncrimping of collagen

44 fibres and the contribution of proteoglycans in the ligament extracellular matrix to intra-fibrillar

45 gliding, which results in more tissue elongations and higher energy dissipation. 
46 This study further expands our understanding of canine CCL behaviour, providing data for

47 material models of femur-CCL-tibia complexes, and demonstrating the challenges for

48 engineering complex biomaterials such as knee joint ligaments. 


\section{Introduction}

50 Ligaments play a major role in stifle (knee) joint stability (Budras, 2007; Levangie and Norkin,

51 2005), with part of the primary support being provided by the cranial cruciate ligament (CCL)

52 (Carpenter and Cooper, 2000; Slatter, 2002). The CCL is the most commonly ruptured canine

53 stifle joint ligament (CCLR) (Arnoczky, 1988; Gianotti et al., 2009) following acute injury or

54 chronic disease, which can lead to destabilisation of surrounding structures and the subsequent

55 development of osteoarthritis (Arnoczky, 1988; Bennett et al., 1988; Brooks, 2002; Comerford et

56 al., 2006). There is a large economic cost associated with managing canine CCLR, for example

57 in the United States alone the economic cost was estimated to be at least one billion dollars in

582003 (Wilke et al., 2005). Both human and canine CCL failure significantly increases the

59 incidence of age-associated joint degeneration (Lee et al., 2014; Liu et al., 2003; Peters et al.,

60 2018) and so understanding the tissue's fundamental material properties can assist with the

61 prediction and effective management of ligament injuries.

62 The phenomenon of viscoelastic characteristics including strain rate dependency, hysteresis, creep and stress relaxation has been observed consistently in soft biological tissues such as the sclera (Elsheikh et al., 2010; Geraghty et al., 2020), cornea (Elsheikh et al., 2011; Kazaili et al., 2019), and tendon (Robinson et al., 2004; Zuskov et al., 2020). Similar to the other biological tissues, ligaments inherit viscoelastic characteristics meaning they exhibiting both elastic and viscous behaviour, hence they are history- and time-dependent (Bonner et al., 2015; Fung, 1993; Ristaniemi et al., 2018). The initial part of the non-linear load-deformation behaviour in ligaments is the toe region where the wavy collagen fibres become taut and straighten as load is applied, hence the crimp is removed (Fratzl et al., 1998). In this zone, there is a relatively large deformation of the tissue with little increase in load and this permits initial joint deformations 
72 with minimal tissue resistance (Amis, 2004; Dale and Baer, 1974; Fratzl et al., 1998; Wingfield 73 et al., 2000) (Fig. 1).

74 Several studies tested for material characteristics of knee joint ligaments at traumatic loading 75 rates and to failure loads (Crisco et al., 2002; Crowninshield and Pope, 1976; Lydon et al., 1995).

76 The loading rate is reported to be directly proportional to the tension which develops in

77 ligaments (Pioletti et al., 1999; Woo et al., 1990b). This characteristic was also evident in a study 78 investigating lower strain rates between approximately 2 and $54 \% /$ min representing 79 physiological conditions other than trauma (Haut and Little, 1969). The study reported no change 80 in the overall shape of the stress-strain curve, however, rapid change in the tangent modulus was 81 found with the slow strain rates (between 1.7 and $10.8 \% / \mathrm{min}$ ) and the change became

82 progressively smaller with higher strain rates (above $10.8 \% / \mathrm{min}$ ). Similarly, it was reported that 83 strain rate dependency decreases with the increase of deformation rate (Bonner et al., 2015;

84 Crisco et al., 2002). The stress-strain behaviour in the toe region (6\% strain) showed strain rate 85 dependency in canine CCL (Haut and Little, 1969). However, a study on rabbit medial collateral 86 ligament complexes at varying strain rates (between 0.66 and $9300 \% / \mathrm{min}$ ) showed that the

87 ligaments were only minimally strain rate dependent (Woo et al., 1990b, 1981). The small effect 88 of strain rate stiffening could be because the studies combined stress-strain characteristics at the 89 toe region with the elastic region (Haut and Little, 1969; Ristaniemi et al., 2018).

90 During high strain rates, hysteresis (energy dissipated) in the ligament may protect the tissue

91 from injury (Bonifasi-Lista et al., 2005). However, there are contradicting findings about

92 hysteresis in soft biological tissues in relation to strain rates. Initially, hysteresis was believed to

93 be weakly dependent on strain rates (Fung, 1993). In contrary, a study on the viscoelastic tensile

94 response of bovine cornea showed an increase in hysteresis with decreasing strain rates (Boyce et 
95 al., 2007). It is suggested that Fung's belief in this phenomenon was based on a small number of

96 experiments on rabbit papillary muscle using only three different strain rates (Haslach, 2005).

97 Hence, Fung's findings only approximately support the independence of hysteresis from strain

98 rates.

99 Therefore, there is a lack of understanding on the strain rate dependency and hysteresis of canine

100 CCLs. Current information is limited to no clear methodological investigations on the strain rate

101 dependency and hysteresis of the CCLs at the toe region (the initial part of non-linear load-

102 deformation behaviour) where collagen fibres tighten and uncrimps with applied load, and

103 importantly, any alterations in the extracellular matrix will be observed (Comerford et al., 2014;

104 Lujan et al., 2009). Therefore, the purpose of this study was to characterise the viscoelastic

105 properties of healthy canine CCLs as a femur-CCL-tibia complex, with a focus on the toe region

106 of the stress-strain properties. This quantification is important when comparing the mechanical

107 characteristics of the CCL and when developing synthetic, auto and allo-grafts to be used in

108 future therapies for ligament replacement.

109

110 Materials \& Methods

111 Cranial cruciate ligament storage and preparation

112 Cadaveric disease-free canine stifle joint pairs from skeletally mature Staffordshire bull terriers

113 ( $\mathrm{n}=6$ pairs) euthanatized for reasons other than musculoskeletal injury were obtained with full

114 ethical permission from the Veterinary Research Ethics Committee ((VREC65), Institute of

115 Veterinary Science, University of Liverpool). Inclusion criteria for cadaveric samples were a

116 bodyweight $>15 \mathrm{~kg}$ and age between 1.5 and 5 years old. The entire stifle joints were frozen at - 
$11720^{\circ} \mathrm{C}$ until required and defrosted at room temperature prior to removing the CCLs as a femur-

118 CCL-tibia complex (Readioff, 2017; Readioff et al., 2020). In order to harvest the femur-CCL-

119 tibia complex, initially the stifle joints were dissected. Subsequently, approximately $10 \mathrm{~mm}$ of

120 the femoral and tibial bones were left connected to the CCLs which allowed for the measurement

121 of end-to-end ligament deformation as well as helping to facilitate the clamping of the specimen

122 (Fig. 2 and 3).

123 The extracted femur-CCL-tibia complexes were maintained in a moistened state in paper towels

124 soaked with phosphate buffered saline (PBS; Sigma, Poole, UK) and frozen at $-80^{\circ} \mathrm{C}$ until they

125 were required for testing (Woo et al., 1986). Prior to testing, the samples were thawed at room

126 temperature and two $1.1 \mathrm{~mm}$ arthrodesis wires (Veterinary Instrumentation, Sheffield, UK) were

127 drilled through the tibial and femoral bone ends (Fig. 2). These pins were placed to provide extra

128 grip as well as to replicate the ligament's slight proximal-to-distal outward spiral when secured

129 using custom-built steel clamps (Arnoczky, 1983; Arnoczky and Marshall, 1977). The ducktail

130 clamps were designed to provide a secure grip as well as ensuring that the CCLs were free and

131 unobstructed throughout the experiment (Fig. 3). The clamped samples were then mounted on a

132 mechanical testing machine.

\section{Cranial cruciate ligament length}

135 A modified version of a previously described method was used to determine the average length

136 of CCL from the craniomedial and caudolateral portions of a ligament (Comerford et al., 2005;

137 Vasseur et al., 1991). In this study, measurements between the insertion and origin of the CCLs

138 at the cranial and caudal planes, as well as the lateral and medial planes were taken using a 
139 Vernier callipers (D00352, Duratool, Taiwan) accurate to $\pm 10 \mu \mathrm{m}$. The mean values of these four

140 length measurements were recorded to give an accurate record of the length of the CCL before

141 deformation (Supplementary Materials (Fig. S1)).

142

143 Cranial cruciate ligament cross-sectional area

144 The method by Goodship and Birch was used to measure cross sectional area (CSA) of the CCLs

145 (Goodship and Birch, 2005). In brief, alginate dental impression paste (UnoDent, UnoDent Ltd.,

146 UK) was used to make a mould around the CCL. Once set, the mould was removed from the

147 CCL and was used to create replicas of the CCLs. The replicas were cut into two in the middle

148 and the surface of the replicas showing middle CSA were estimated using ImageJ (a public

149 domain Java image processing program) (Supplementary Materials (Fig. S2)).

150

151 Mechanical testing

152 An Instron 3366 materials testing machine (Instron, Norwood, MA) fitted with a $10 \mathrm{~N}$ load cell

153 (Instron 2530-428 with $\pm 0.025 \mathrm{~N}$ accuracy) was used to perform tensile tests. Initially, a preload

154 of $0.1 \mathrm{~N}$ was applied to remove laxity within the CCL (Provenzano et al., 2002). Application of

155 the preload was then followed by preconditioning the CCLs to ensure that they were in a steady

156 state and would produce comparable and reproducible load-elongation curves (Butler et al.,

157 1978; Fung, 1993; Savelberg et al., 1993). Preconditioning involved performing ten loading-

158 unloading cycles up to a maximum load of $10 \mathrm{~N}$ at $10 \% / \mathrm{min}$ strain rate (Ebrahimi et al., 2019;

159 Woo et al., 1991). Subsequently the CCL was subjected to cyclic tensile loading-unloading tests

160 investigating stress-strain behaviour of the ligament at the toe region through the application of 
$16110 \mathrm{~N}$ load at sequential slow strain rates of $0.1,1$ and $10 \% / \mathrm{min}$. Each strain rate consisted of

162 three loading-unloading cycles which allowed for reproducible results. Between each two cycles,

163 including the preconditioning procedure, a period of six minutes recovery time was given

164 (Ebrahimi et al., 2019; Viidik, 1968). From the paired stifle joints, the left CCLs were exposed to

165 an ascending strain rate test in which the rate of strain was increased from 0.1 to 1 and to 10

$166 \% / \mathrm{min}$ and the right CCLs were exposed to a descending strain rate in which the CCL was tested

167 under decreasing strain rates from 10 to 1 and to $0.1 \% /$ min (Pioletti et al., 1999; Pioletti and

168 Rakotomanana, 2000). A slow speed was chosen to better observe tissue response to loading at

169 the toe region of load-deformation curves. The reverse orders of strain rate tests (ascending and

170 descending strain rates tests) were carried out to identify characteristics associated with strain

171 history of the ligaments at the toe region.

172

173 Data Analysis

174 Analyses on the collected load-deformation data were performed using Microsoft Excel

175 spreadsheets (Microsoft Office 2010, US) and MATLAB (MATLAB R2020a) (code for the

176 analysis and graphs can be found in the Supplementary Materials). Nominal stress and strain

177 values were estimated following Equations 1 and 2 (Haut and Little, 1969; Woo et al., 1981) and

178 from these stress and strain values, tangent modulus values were determined (Equation 3).

179 Numerical integrations (the trapezoidal rule) on the stress-strain curves were used to estimate the

180 stored energy in the ligaments during loading and unloading tests (Equation 4). The hysteresis

181 was then calculated from the difference between the stored energy during loading and unloading

182 tests (Elsheikh et al., 2008) (Equation 5). In addition, ligament extension before and after 
183 recovery were studied to investigate strain history and strain rate dependencies as a result of

184 applying loads at different strain rate orders (loading at ascending or descending rates).

$$
\sigma=\frac{F}{C S A}
$$

where $\sigma$ is stress in MPa, $F$ is applied load in $\mathrm{N}$ and CSA is cross-sectional area at the middle of the CCL in $\mathrm{mm}^{2}$.

$\varepsilon=\frac{\Delta L}{L_{0}}$

where $\varepsilon$ is strain, $\Delta L$ is change in length in $\mathrm{mm}\left(\Delta L=L_{0}-L_{1}\right), L_{0}$ is initial length and $L_{1}$ is deformed length of the CCL in mm.

$E_{\text {tan }}=\frac{\delta \sigma}{\delta \varepsilon}$

Equation 3

where $E_{\text {tan }}$ is tangent modulus in $\mathrm{MPa}$.

$U=\sum_{k=1}^{N} \frac{1}{2} \times\left(\sigma_{k-1}+\sigma_{k}\right) \times \Delta \varepsilon_{k}$

Equation 4

where $U$ is the stored energy in $\mathrm{MPa}, N$ is the resolution of the trapezoidal partition, and $\Delta$ $\varepsilon_{k}$ is the length of the $k^{t h}$ subinterval $\left(\Delta \varepsilon_{k}=\varepsilon_{k}-\varepsilon_{k-1}\right)$. 
Hysteresis $=U_{\text {Loading }}-U_{\text {Unloading }}$

Equation 5

where $U_{\text {Loading }}$ and $U_{\text {Loading }}$ represent the stored energy during loading and unloading of the ligaments in MPa.

185

186

Statistical Analysis

187 CCL lengths measured at different planes were categorised into cranial, caudal, medial and

188 lateral groups. Statistical tests were performed using one-way analysis of variance (ANOVA)

189 followed by a Bonferroni post-hoc test for multiple comparisons.

190 A two-tailed t-test (two samples with unequal variance) was used to test for differences between

191 results obtained from the ascending and descending strain rate tests. In addition, one-way

192 ANOVA followed by a Bonferroni post-hoc test for multiple comparisons was performed to test

193 dependencies of tensile responses of the ligaments on strain rates. Distribution of data was

194 illustrated in boxplots and suspected outliers were defined as any value greater than or equal to

1951.5 times the interquartile range (range between the first and third quartiles).

196 All statistical analyses were performed in Microsoft Office Excel and 95\% confidence level ( $p$ $197<0.05$ ) was selected to define significance for all statistical tests.

198

199 Results

200 Cranial cruciate ligament samples 
201 The CCL samples ( $\mathrm{n}=6$ paired stifle joints) used to investigate mechanical properties of the

202 ligament were of mixed gender (female $=3$ and male $=3$ ) and the bodyweight of the cadavers were

203 in the range of 17 to $25.5 \mathrm{~kg}(20.68 \pm 3.85 \mathrm{~kg})$.

204

205 Cranial cruciate ligament length

206 The lengths of the CCLs at different planes were in the range of 7.88 to $23.16 \mathrm{~mm}$ and the

207 measurements at different planes of the individual ligaments are reported in Table 1.

208 The ANOVA test showed statistically significant results in measuring CCL length in different

209 plane views. The analysis showed that length measurements recorded at different planes were

210 statistically different except for comparisons between medial and lateral planes (Supplementary

211 Material (Table S1)).

212

213 Cranial cruciate ligament cross-sectional area

214 The cross-sectional areas of the CCLs were in the range of 11.09 to $23.62 \mathrm{~mm}^{2}\left(16.1 \pm 5.1 \mathrm{~mm}^{2}\right)$

215 and cross-sectional areas of individual ligaments are reported in Table 2.

216

217 Mechanical properties

218 Stress-strain

219 The stress-strain curves at $0.1,1$ and $10 \% /$ min strain rates conformed to the typical non-linear

220 behaviour as expected in canine CCLs (Haut and Little, 1969) (Fig. 4a). The stress-strain curves 
221 illustrated an increase in stress with increasing strain, and similarly an increase in stiffness was

222 observed with increasing strain rates (Fig. 4b) (Supplementary Materials (Fig. S6)). Although

223 there was a small increase in stress with increasing strain rates, the increase was not statistically

224 significant.

225 The stress responses of the ligaments during ascending test protocol, where the cyclic loading 226 commenced with $0.1 \% / \mathrm{min}$ then increased to $1 \% / \mathrm{min}$ and finally to $10 \% / \mathrm{min}$, were similar to

227 responses during descending test protocol (the reverse of ascending test protocol). The stress-

228 strain curves show that there are minimal differences in stress values between the two test

229 protocols below 3\% strain, and these differences become more distinguishable above $3 \%$ strain

230 (Fig. 5a, b and c). The testing protocols only minimally affected the stress-strain characteristics

231 and not statistically significant. There are notably different mechanical behaviours among the

232 specimens, as indicated by the grey dots in Fig. 5a, b and c, and not all specimens reached 5\%

233 strain (Supplementary Materials (Fig. S3, S4 and S5).

235 Tangent modulus-stress

236 Tangent modulus $\left(E_{t}\right)$, indicating the stiffness behaviour of the CCLs, increased with increasing 237 stress (Fig. 4b) in both ascending and descending testing protocols. Similar to the observations

238 from the stress-strain curves, the tangent modulus-stress lines between the two testing protocols

239 (ascending and descending) were only minimally different and not statistically significant.

240 Although not statistically significant, the increase in tangent modulus with strain rates was

241 notable at higher stress values (Fig. 5d, e and f). For example, at $0.5 \mathrm{MPa}$ stress, average tangent 
242 modulus values from the ascending test protocol were 26.62, 31.40 and $32.66 \mathrm{MPa}$ during

243 loading at $0.1,1$ and $10 \% /$ min strain rates.

244

245 Hysteresis

246 The results of this study show that hysteresis or dissipated energy are statistically different

247 between the ascending and descending testing protocols $(p=0.0043)$. The mean values for

248 hysteresis at $0.1,1$ and $10 \% /$ min strain rates were 0.0032 (cycle 13), 0.0020 (cycle 16) and

2490.0016 (cycle 19) MPa in ascending and 0.0040 (cycle 19), 0.0042 (cycle 16) and 0.0037 (cycle

250 13) $\mathrm{MPa}$ in descending testing protocol (Fig. 6a). The dissipated energy decrease from the first

251 preconditioning cycle to the tenth (last) cycle was $85 \%$ for both testing protocols. In addition,

252 hysteresis decreased with increasing strain rates (Fig. 6b). This characteristic was statistically

253 significant during the ascending testing protocol $(p=0.039$ between 0.1 and $1 \% / \mathrm{min}$, and $p=$

2540.013 between 0.1 and $10 \% / \mathrm{min})$. The metadata of hysteresis can be found in Supplementary

255 Material (Table S2).

256

257 Recovery

258 Length of the CCLs before and after the recovery period between each cycle showed consistent

259 values during preconditioning and these values were in the range of 0.095 to $0.078 \mathrm{~mm}$ during

260 ascending and 0.124 to $0.095 \mathrm{~mm}$ during descending tests (Fig. 7a). Unlike hysteresis, statistical

261 analysis showed that tissue recovery was not different during ascending and descending tests.

262 However, tissue length recovery was strain rate dependent and statistical analysis showed 
263 differences in recovery between 0.1 and $1 \% / \min (p=0.018)$, and 0.1 and $10 \% / \min (p=0.001)$

264 (Fig. 7b).

265

266 Discussion

267 The aim of this study was to gain a greater understanding of the viscoelastic behaviour of the

268 canine CCLs as a femur-CCL-tibia complex at the toe region of the stress-strain curves in order

269 to better mechanically detail the material for its use in developing future therapies. Therefore, we

270 carried out an experimental study investigating the nonlinear viscoelastic properties of CCLs,

271 namely strain rate dependency, hysteresis and recovery, from healthy canine stifle joints. The

272 findings in this study are the first to report the slow strain rate dependency of the canine cruciate

273 ligament across three orders of magnitude with ascending and descending test arrangements. A

274 previous study showed that with high strain rates, the toe region of stress-strain curves appears at

275 lower strain levels (Haut and Little, 1969), however in order to study the toe region in detail

276 without being limited to the level of strain, slow strain rates $(\leq 10 \% / \mathrm{min})$ were utilised during

277 mechanical tests in the current study.

278 Similar to previous studies, the measured CCL length and cross-sectional area were used in the

279 calculations for stress and strain values (Wingfield et al., 2000). The difference in the CCLs

280 length in medial and lateral planes was smaller than other planes, and this could be due to the

281 anatomical structure of the femur-CCL-tibia complex.

282 The non-linear stress-strain pattern for the CCLs is consistent to that previously reported in

283 studies on biological tissues such as tendons and ligaments (Bonner et al., 2015; Crisco et al., 
284 2002; Haut and Little, 1969; Pioletti et al., 1999; Pioletti and Rakotomanana, 2000). The stress-

285 strain and tangent modulus-stress characteristics of the CCLs were similar during the ascending

286 and descending testing protocols (Fig. 4 and 5). These findings are similar to a previous study on

287 bovine anterior cruciate ligament-bone complex where specimens were loaded up to $300 \mathrm{~N}$ at

288 seven different strain rates $(6,60,300,600,1200,1800$ and $2400 \% / \mathrm{min})$ and then tested for

289 strain rate order by reloading the ligaments at the 6 and $300 \% / m i n$ strain rates (Pioletti et al.,

290 1999). They found identical stress-strain behaviour for the initial and reloaded specimens

291 suggesting no difference in changing test protocols via strain rate orders. Their study applied

292 higher strain rates $(6-2400 \% / \mathrm{min})$ than those used in the current paper and they reloaded the

293 tissue in an ascending strain rate order only. Pioletti et al. did not study tissue hysteresis or

294 recovery, but they reported increases in linear tangent moduli with increasing strain rates

295 (although not statistically significant) which is similar to the findings in the current study.

296 The mechanical response of human knee ligaments to loading depends on strain rate which is

297 less pronounced at lower rates (Dorlot et al., 1980; van Dommelen et al., 2005) and this was also

298 observed in the current study. It is believed that during lower strain rates the collagen fibrils in

299 patella tendons undergo significantly less recruitment (Clemmer et al., 2010) and this could

300 potentially be similar in the case of the CCLs. At slow strain rates $(\leq 10 \% / \mathrm{min})$ the collagen

301 fibrils uncrimp with applied load and then show intra-fibrillar gliding (Bonner et al., 2015;

302 Karunaratne et al., 2018). However, at fast strain rates ( $\geq 300 \% / \mathrm{min})$ fibrils go from an unloaded

303 state directly to intra-fibrillar gliding where the matrix bond between the collagen molecules are

304 broken before the removal of collagen crimps (Bonner et al., 2015). This could mean that the

305 extracellular matrix components such as proteoglycans, which is directly linked to the mechanics 
306 of ligaments during uncrimping of the collagen fibres, might not affect the mechanical response

307 during loads at higher strain rates.

308 In our study, hysteresis, which represents the dissipation of energy within the tissue, has shown

309 some dependencies on strain rates and decreases with increasing strain rates (Fig. 6b). This

310 finding contradicts the conclusions from previous literature (Bonifasi-Lista et al., 2005; Woo et

311 al., 1981) but agrees with a recent study on tendon fascicle mechanics (Rosario and Roberts,

312 2020) and a study on bovine cornea (Boyce et al., 2007) which found a decrease in strain energy

313 storage with increased loading rate. The discrepancies in results might be due to following

314 different testing protocols, in particular the rate of applied loads. In the current study, where slow

315 strain rates of $\leq 10 \% / \mathrm{min}$ were used, the tissue goes through more steps (uncrimping collagen

316 fibres, intra-fibrillar gliding and then loading of collagen respectively with applied loads) during

317 lower strain rates (Bonner et al., 2015), and this slow process results in more tissue elongations

318 hence higher energy dissipation. Energy dissipation was highest during the first precondition

319 cycle and this could be a result of tissue handling and loading history of the CCLs (Fig. 6). In

320 addition, it is possible that the ligament was dissipating higher energy at the initial loading cycles

321 (during preconditioning) because of microstructural reorganisation. Similar to previous literature,

322 this study found a decrease in hysteresis during preconditioning cycles (Woo et al., 1986; Yahia

323 and Drouin, 1990).

324 The CCLs showed higher length recovery during higher strain rates compared to the lower strain

325 rates (Fig. 7). This behaviour could be a result of resilience in the fascicular level of the tissue. It

326 has been reported that during slow strain rates, the resilience in the fascicular level is lowest

327 (Rosario and Roberts, 2020) which could lead to higher changes in the microstructural

328 organisation of the tissue. Hence, the tissue's length might not fully recover within the same 
329 recovery time as during higher strain rate tests. However, it is important to note that although the

330 higher strain rate might seem to result in a more recovered ligament length, it is possible that the

331 tissue collagen fibres are still crimping back as a result of previous loading history or insufficient

332 recovery time. Further study in this area especially at the microstructural level of knee ligaments

333 is necessary to better understand the effects of loading rates on the organisations of the fibres and

334 extracellular matrix.

335 There were several limitations to our study. Preparing specimens for mechanical tests as a whole

336 unit (femur-CCL-tibia complex) might have introduced some limitations such as overlooking the

337 complexity of the anatomical structure of the CCLs which consists of two fibre bundles

338 (caudolateral (CLB) and craniomedial bands (CMB)) functioning independently from one

339 another in stifle joint flexion and extension (Arnoczky and Marshall, 1977; Carpenter and

340 Cooper, 2000). Independent functioning of the CLB and the CMB allows the fibre bundles to

341 reach their maximum potential (Arnoczky and Marshall, 1977; Carpenter and Cooper, 2000;

342 Tanegashima et al., 2019). However, it is important to note that these two fibre bundles are not

343 structurally segregated within the tissue, thus allowing the ligament to function as a united

344 structure (Heffron and Campbell, 1978). In addition, the approximation methods adopted to

345 measure the cross-sectional area and length of the specimens might be considered as another

346 limitation. Further investigation with a larger number of specimens might improve the reliability

347 of the statistical analysis and provide a broader view on the effect of cadaveric demography (i.e.

348 age, gender, bodyweight) on the mechanical properties and microstructural organisations of knee

349 ligaments (Duval et al., 1999; Woo et al., 1990a, 1990b). In addition, investigating material

350 properties of the ligaments using other quantitative measures such as stress relaxation or creep

351 could further expand our knowledge on the viscoelasticity of the tissue (Amis, 1985). 


\section{Conclusions}

354 The current study focused on the viscoelastic behaviour, such as strain rate dependency,

355 hysteresis and recovery of canine CCLs at slow strain rates to better understand the tissue

356 behaviour at the toe region where the constituents of the extracellular matrix makes a major

357 contribution to ligament mechanics.

358 Our changing test protocols via strain rate orders only affected hysteresis which might be a result

359 of the strain history of the tissue or high-level of biological variability across samples (Gardiner

360 and Weiss, 2003; Harris et al., 2016). The stress-strain of the CCLs at the toe-region associated

361 with the extracellular matrix of the ligaments was not strain rate dependent. However, hysteresis

362 and recovery were strain rate dependent and this is likely due to changes in microstructural

363 organisation of the ligaments during mechanical tests.

364 The result of our study indicates the need for further investigations on the viscoelastic behaviour

365 of the canine CCLs when loaded with different orders of strain rates, with a focus on

366 extracellular matrix and collagen fibre organisations.

\section{Acknowledgments}

369 We thank Mr. Lee Moore, Mr. Ben Jones and the staff at Veterinary Teaching Suite, School of

370 Veterinary Science for their assistance during sample collection. We also thank Mr. John Curran

371 at School of Engineering, University of Liverpool, for their assistance during manufacturing

372 parts of the experimental setup. 
374 References

375 Amis, A.A., 2004. The Biomechanics of Ligaments, in: Poitout, D.G. (Ed.), Biomechanics and

376 Biomaterials in Orthopedics. Springer, London, pp. 550-563. https://doi.org/10.1007/978-1-

377 4471-3774-0_48

378 Amis, A.A., 1985. Biomechancis of Ligaments, in: Ligament Injuries and Their Treatment.

379 Chapman and Hall Ltd, London, pp. 3-25.

380 Arnoczky, S.P., 1988. The cruciate ligaments: The enigma of the canine stifle. J. Small Anim.

381 Pract. 29, 71-90. https://doi.org/DOI 10.1111/j.1748-5827.1988.tb02267.x

382 Arnoczky, S.P., 1983. Anatomy of the anterior cruciate ligament. Clin. Orthop.

383 Arnoczky, S.P., Marshall, J.L., 1977. The cruciate ligaments of the canine stifle: An anatomical 384 and functional analysis. Am. J. Vet. Res. 38, 1807-1814.

385 Bennett, D., Tennant, B., Lewis, D.G., Baughan, J., May, C., Carter, S., 1988. A reappraisal of 386 anterior cruciate ligament disease in the dog. J. Small Anim. Pract. 29, 275-297.

387 https://doi.org/DOI 10.1111/j.1748-5827.1988.tb02286.x

388 Bonifasi-Lista, C., Lake, S.P., Small, M.S., Weiss, J.A., 2005. Viscoelastic properties of the

389 human medial collateral ligament under longitudinal, transverse and shear loading. J Orthop Res.

390 https://doi.org/10.1016/j.orthres.2004.06.002 
391 Bonner, T.J., Newell, N., Karunaratne, A., Pullen, A.D., Amis, A.A., A, M.J.B., Masouros, S.D.,

392 2015. Strain-rate sensitivity of the lateral collateral ligament of the knee. J. Mech. Behav.

393 Biomed. Mater.

394 Boyce, B.L., Jones, R.E., Nguyen, T.D., Grazier, J.M., 2007. Stress-controlled viscoelastic

395 tensile response of bovine cornea. J. Biomech. 40, 2367-2376. https://doi.org/DOI

396 10.1016/j.jbiomech.2006.12.001

397 Brooks, P.M., 2002. Impact of osteoarthritis on individuals and society: How much disability?

398 Social consequences and health economic implications. Curr. Opin. Rheumatol.

399 https://doi.org/10.1097/00002281-200209000-00017

400 Budras, K.-D., 2007. Anatomy of the dog, 5th ed, Vet series. Schlütersche, Hannover.

401 Butler, D.L., Noyes, F.R., Grood, E.S., 1978. Measurement of the mechanical properties of

402 ligaments, in: CRC Handbook of Engineering in Medicine and Biology Section B: Instruments

403 and Measurements. CRC Press Inc., West Palm Beach, pp. 279-314.

404 Carpenter, D.H., Cooper, R.C., 2000. Mini review of canine stifle joint anatomy. Anat. Histol.

405 Embryol. 29, 321-329. https://doi.org/DOI 10.1046/j.1439-0264.2000.00289.x

406 Clemmer, J., Liao, J., Davis, D., Horstemeyer, M.F., Williams, L.N., 2010. A mechanistic study

407 for strain rate sensitivity of rabbit patellar tendon. J. Biomech. 43, 2785-2791.

408 https://doi.org/10.1016/j.jbiomech.2010.06.009

409 Comerford, E.J., Geraghty, B., Hama Rashid, R., Elsheikh, A., 2014. The Contribution of

410 Proteoglycans to the Viscoelasticity of the Canine Anterior Cruciate Ligament. Osteoarthritis

411 Cartilage. 
412 Comerford, E.J., Tarlton, J.F., Innes, J.F., Johnson, K.A., Amis, A.A., Bailey, A.J., 2005.

413 Metabolism and composition of the canine anterior cruciate ligament relate to differences in knee

414 joint mechanics and predisposition to ligament rupture. J. Orthop. Res. 23, 61-66.

415 https://doi.org/10.1016/j.orthres.2004.05.016

416 Comerford, E.J., Tarlton, J.F., Wales, A., Bailey, A.J., Innes, J.F., 2006. Ultrastructural

417 differences in cranial cruciate ligaments from dogs of two breeds with a differing predisposition

418 to ligament degeneration and rupture. J Comp Pathol. https://doi.org/10.1016/j.jcpa.2005.06.004

419 Crisco, J.J., Moore, D.C., McGovern, R.D., 2002. Strain-rate sensitivity of the rabbit MCL

420 diminishes at traumatic loading rates. J. Biomech. 35, 1379-1385.

421 Crowninshield, R.D., Pope, M.H., 1976. The strength and failure characteristics of rat medial 422 collateral ligaments. J. Trauma 16, 99-105.

423 Dale, W.C., Baer, E., 1974. Fiber-buckling in composite systems: A model for ultrastructure of

424 uncalcified collagen tissues. J. Mater. Sci. 9, 369-382. https://doi.org/Doi 10.1007/Bf00737836

425 Dorlot, J.M., Ait Ba Sidi, M., Tremblay, G.M., Drouin, G., 1980. Load elongation behavior of

426 the canine anterior cruciate ligament. J. Biomech. Eng. https://doi.org/10.1115/1.3149572

427 Duval, J.M., Budsberg, S.C., Flo, G.L., Sammarco, J.L., 1999. Breed, sex, and body weight as

428 risk factors for rupture of the cranial cruciate ligament in young dogs. J. Am. Vet. Med. Assoc. $429215,811-814$.

430 Ebrahimi, M., Mohammadi, A., Ristaniemi, A., Stenroth, L., Korhonen, R.K., 2019. The effect 431 of different preconditioning protocols on repeatability of bovine ACL stress-relaxation response 
432 in tension. J. Mech. Behav. Biomed. Mater. 90, 493-501.

433 https://doi.org/10.1016/j.jmbbm.2018.10.041

434 Elsheikh, A., Geraghty, B., Alhasso, D., Knappett, J., Campanelli, M., Rama, P., 2010. Regional

435 variation in the biomechanical properties of the human sclera. Exp. Eye Res. 90, 233-624.

436 https://doi.org/10.1016/j.exer.2010.02.010

437 Elsheikh, A., Kassem, W., Jones, S.W., 2011. Strain-rate sensitivity of porcine and ovine 438 corneas. Acta Bioeng. Biomech. 13, 25-36.

439 Elsheikh, A., Wang, D., Rama, P., Campanelli, M., Garway-Heath, D., 2008. Experimental 440 assessment of human corneal hysteresis. Curr. Eye Res. 33, 205-213.

441 https://doi.org/10.1080/02713680701882519

442 Fratzl, P., Misof, K., Zizak, I., Rapp, G., Amenitsch, H., Bernstorff, S., 1998. Fibrillar structure 443 and mechanical properties of collagen. J. Struct. Biol. 122, 119-122. https://doi.org/DOI $444 \quad 10.1006 /$ jsbi.1998.3966

445 Fung, Y.C., 1993. Biomechanics: Mechanical properties of living tissues, 2nd ed. Springer, New 446 York.

447 Gardiner, J.C., Weiss, J.A., 2003. Subject-specific finite element analysis of the human medial 448 collateral ligament during valgus knee loading. J. Orthop. Res. Off. Publ. Orthop. Res. Soc. 21, 449 1098-1106. https://doi.org/10.1016/S0736-0266(03)00113-X

450 Geraghty, B., Abass, A., Eliasy, A., Jones, S.W., Rama, P., Kassem, W., Akhtar, R., Elsheikh, 451 A., 2020. Inflation experiments and inverse finite element modelling of posterior human sclera.

452 J. Biomech. 98, 109438. https://doi.org/10.1016/j.jbiomech.2019.109438 
453 Gianotti, S.M., Marshall, S.W., Hume, P.A., Bunt, L., 2009. Incidence of anterior cruciate

454 ligament injury and other knee ligament injuries: A national population-based study. J. Sci. Med.

455 Sport 12, 622-627. https://doi.org/10.1016/j.jsams.2008.07.005

456 Goodship, A.E., Birch, H.L., 2005. Cross sectional area measurement of tendon and ligament in

457 vitro: a simple, rapid, non-destructive technique. J. Biomech. 38, 605-608. https://doi.org/DOI

458 10.1016/j.jbiomech.2004.05.003

459 Harris, M.D., Cyr, A.J., Ali, A.A., Fitzpatrick, C.K., Rullkoetter, P.J., Maletsky, L.P., Shelburne,

460 K.B., 2016. A Combined Experimental and Computational Approach to Subject-Specific

461 Analysis of Knee Joint Laxity. J. Biomech. Eng. 138. https://doi.org/10.1115/1.4033882

462 Haslach, H.W., 2005. Nonlinear viscoelastic, thermodynamically consistent, models for

463 biological soft tissue. Biomech. Model. Mechanobiol. 3, 172-189.

464 https://doi.org/10.1007/s10237-004-0055-6

465 Haut, R.C., Little, R.W., 1969. Rheological properties of canine anterior cruciate ligaments. J.

466 Biomech. 2, 289-298. https://doi.org/Doi 10.1016/0021-9290(69)90085-2

467 Heffron, L.E., Campbell, J.R., 1978. Morphology, histology and functional anatomy of the 468 canine cranial cruciate ligament. Vet. Rec.

469 Karunaratne, A., Li, S., Bull, A.M.J., 2018. Nano-scale mechanisms explain the stiffening and 470 strengthening of ligament tissue with increasing strain rate. Sci. Rep.

471 https://doi.org/10.1038/s41598-018-21786-Z 
472 Kazaili, A., Geraghty, B., Akhtar, R., 2019. Microscale assessment of corneal viscoelastic

473 properties under physiological pressures. J. Mech. Behav. Biomed. Mater. 100, 103375.

474 https://doi.org/10.1016/j.jmbbm.2019.103375

475 Lee, K., Williamson, K., Clegg, P., Comerford, E., Canty-Laird, E., 2014. The stem cell niche in 476 tendon and ligament: investigating alterations with ageing and disease, in: British Journal of 477 Sports Medicine. BMJ, pp. A35-A36. https://doi.org/10.1136/bjsports-2014-094114.54

478 Levangie, P.K., Norkin, C.C., 2005. Joint structure and function: A comprehensive analysis, 4th 479 ed. F.A. Davis Co., Philadelphia.

480 Liu, W.H., Burton-Wurster, N., Glant, T.T., Tashman, S., Sumner, D.R., Kamath, R.V., Lust, G., 481 Kimura, J.H., Cs-Szabo, G., 2003. Spontaneous and experimental osteoarthritis in dog:

482 similarities and differences in proteoglycan levels. J. Orthop. Res. https://doi.org/Doi $483 \quad 10.1016 / \mathrm{S} 0736-0266(03) 00002-0$

Lujan, T.J., Underwood, C.J., Jacobs, N.T., Weiss, J.A., 2009. Contribution of glycosaminoglycans to viscoelastic tensile behavior of human ligament. J. Appl. Physiol.

486 https://doi.org/10.1152/japplphysiol.90748.2008

Lydon, C., Crisco, J.J., Panjabi, M., Galloway, M., 1995. Effect of elongation rate on the failure 488 properties of the rabbit anterior cruciate ligament. Clin. Biomech. 10, 428-433. osteoarthritis on the mechanical properties of cartilage and bone in the human knee joint. Sci. 
492 Pioletti, D.P., Rakotomanana, L.R., 2000. Non-linear viscoelastic laws for soft biological tissues.

493 Eur. J. Mech. - ASolids 19, 749-759. https://doi.org/Doi 10.1016/S0997-7538(00)00202-3

494 Pioletti, D.P., Rakotomanana, L.R., Leyvraz, P.F., 1999. Strain rate effect on the mechanical

495 behavior of the anterior cruciate ligament-bone complex. Med. Eng. Phys. 21, 95-100.

496 https://doi.org/10.1016/S1350-4533(99)00028-4

497 Provenzano, P.P., Heisey, D., Hayashi, K., Lakes, R., Vanderby R., Jr., 2002. Subfailure damage

498 in ligament: A structural and cellular evaluation. J. Appl. Physiol. 92, 362-371.

499 Readioff, R., 2017. Viscoelastic Behaviour of the Canine Cranial Cruciate Ligament Complex.

500 University of Liverpool.

501 Readioff, R., Geraghty, B., Comerford, E., Elsheikh, A., 2020. A full-field 3D digital image

502 correlation and modelling technique to characterise anterior cruciate ligament mechanics ex vivo.

503 Acta Biomater. 113, 417-428. https://doi.org/10.1016/j.actbio.2020.07.003

504 Ristaniemi, A., Stenroth, L., Mikkonen, S., Korhonen, R.K., 2018. Comparison of elastic, 505 viscoelastic and failure tensile material properties of knee ligaments and patellar tendon. J.

506 Biomech. 79, 31-38. https://doi.org/10.1016/j.jbiomech.2018.07.031

507 Robinson, P.S., Lin, T.W., Reynolds, P.R., Derwin, K.A., Iozzo, R. V, Soslowsky, L.J., 2004.

508 Strain-rate sensitive mechanical properties of tendon fascicles from mice with genetically

509 engineered alterations in collagen and decorin. J. Biomech. Eng. 126, 252-257.

510 Rosario, M.V., Roberts, T.J., 2020. Loading Rate Has Little Influence on Tendon Fascicle

511 Mechanics. Front. Physiol. 11. https://doi.org/10.3389/fphys.2020.00255 
512 Savelberg, H.H.C.M., Kooloos, J.G.M., Huiskes, R., Kauer, J.M.G., 1993. An Indirect Method to

513 Assess Wrist Ligament Forces with Particular Regard to the Effect of Preconditioning. J.

514 Biomech. 26, 1347-1351. https://doi.org/Doi 10.1016/0021-9290(93)90358-L

515 Slatter, D.H., 2002. Textbook of small animal surgery, 3rd ed. Saunders, Philadelphia.

516 Tanegashima, K., Edamura, K., Akita, Y., Yamazaki, A., Yasukawa, S., Seki, M., Asano, K.,

517 Nakayama, T., Katsura, T., Hayashi, K., 2019. Functional Anatomy of the Craniomedial and

518 Caudolateral Bundles of the Cranial Cruciate Ligament in Beagle Dogs. Vet. Comp. Orthop.

519 Traumatol. VCOT 32, 182-191. https://doi.org/10.1055/s-0039-1678711

520 van Dommelen, J.A.W., Ivarsson, B.J., Jolandan, M.M., Millington, S.A., Raut, M., Kerrigan, 521 J.R., Crandall, J.R., Diduch, D.R., 2005. Characterization of the Rate-Dependent Mechanical 522 Properties and Failure of Human Knee Ligaments. SAE Trans. 114, 80-90.

523 Vasseur, P.B., Stevenson, S., Gregory, C.R., Rodrigo, J.J., Pauli, S., Heitter, D., Sharkey, N.,

524 1991. Anterior cruciate ligament allograft transplantation in dogs. Clin. Orthop. 295-304.

525 Viidik, A., 1968. Elasticity and tensile strength of anterior cruciate ligament in rabbits as

526 influenced by training. Acta Physiol. Scand. 74, 372-380. https://doi.org/DOI 10.1111/j.1748-

527 1716.1968.tb04245.x

528 Wilke, V.L., Robinson, D.A., Evans, R.B., Rothschild, M.F., Conzemius, M.G., 2005. Estimate 529 of the annual economic impact of treatment of cranial cruciate ligament injury in dogs in the 530 United States. J. Am. Vet. Med. Assoc. 227, 1604-1607. 
531 Wingfield, C., Amis, A.A., Stead, A.C., Law, H.T., 2000. Comparison of the biomechanical

532 properties of rottweiler and racing greyhound cranial cruciate ligaments. J. Small Anim. Pract.

533 41, 303-307. https://doi.org/DOI 10.1111/j.1748-5827.2000.tb03206.x

534 Woo, S.L., Orlando, C.A., Camp, J.F., Akeson, W.H., 1986. Effects of postmortem storage by

535 freezing on ligament tensile behavior. J. Biomech. 19, 399-404.

536 Woo, S.L.Y., Gomez, M.A., Akeson, W.H., 1981. The time and history-dependent viscoelastic

537 properties of the canine medical collateral ligament. J. Biomech. Eng. 103, 293-298.

538 Woo, S.L.Y., Hollis, J.M., Adams, D.J., Lyon, R.M., Takai, S., 1991. Tensile properties of the

539 human femur-anterior cruciate ligament-tibia complex. The effects of specimen age and

540 orientation. Am. J. Sports Med. 19, 217-225.

541 Woo, S.L.Y., Ohland, K.J., Weiss, J.A., 1990a. Aging and sex-related changes in the

542 biomechanical properties of the rabbit medial collateral ligament. Mech. Ageing Dev. 56, 129543142.

544 Woo, S.L.Y., Peterson, R.H., Ohland, K.J., Sites, T.J., Danto, M.I., 1990b. The effects of strain

545 rate on the properties of the medial collateral ligament in skeletally immature and mature rabbits:

546 a biomechanical and histological study. J. Orthop. Res. 8, 712-721.

547 https://doi.org/10.1002/jor.1100080513

548 Yahia, L.H., Drouin, G., 1990. Study of the hysteresis phenomenon in canine anterior cruciate

549 ligaments. J. Biomed. Eng.

550 Zuskov, A., Freedman, B.R., Gordon, J.A., Sarver, J.J., Buckley, M.R., Soslowsky, L.J., 2020.

551 Tendon Biomechanics and Crimp Properties Following Fatigue Loading Are Influenced by 
552 Tendon Type and Age in Mice. J. Orthop. Res. Off. Publ. Orthop. Res. Soc. 38, 36-42.

553 https://doi.org/10.1002/jor.24407

554

555 


\section{Table $\mathbf{1}$ (on next page)}

The measured length of cranial cruciate ligaments $(\mathrm{CCL})$ at different measurement planes (cranial, caudal, medial and lateral) for CCLs in paired canine stifle joints ( $n=6$ pairs). 


\begin{tabular}{|c|c|c|c|c|c|c|c|c|c|c|}
\hline \multirow[t]{2}{*}{ No. } & \multicolumn{2}{|c|}{$\begin{array}{c}\text { Cranial Plane } \\
(\mathrm{mm})\end{array}$} & \multicolumn{2}{|c|}{$\begin{array}{c}\text { Caudal Plane } \\
(\mathrm{mm})\end{array}$} & \multicolumn{2}{|c|}{$\begin{array}{c}\text { Medial Plane } \\
(\mathrm{mm})\end{array}$} & \multicolumn{2}{|c|}{$\begin{array}{c}\text { Lateral Plane } \\
(\mathrm{mm})\end{array}$} & \multicolumn{2}{|c|}{$\begin{array}{c}\text { Average } \pm \text { SD } \\
(\mathrm{mm})\end{array}$} \\
\hline & Right & Left & Right & Left & Right & Left & Right & Left & Right & Left \\
\hline 1 & 13.51 & 14.54 & 7.88 & 8.16 & 11.76 & 14.10 & 11.00 & 12.31 & $11.04 \pm 2.35$ & $12.28 \pm 2.91$ \\
\hline 2 & 22.79 & 22.07 & 11.20 & 12.00 & 17.00 & 13.1 & 20.54 & 16.93 & $17.88 \pm 5.05$ & $16.03 \pm 4.55$ \\
\hline 3 & 22.14 & 22.22 & 10.21 & 9.78 & 17.5 & 20.36 & 20.83 & 19.71 & $17.67 \pm 5.34$ & $18.02 \pm 5.59$ \\
\hline 4 & 21.44 & 23.16 & 13.53 & 11.55 & 17.51 & 19.05 & 16.05 & 14.37 & $17.13 \pm 3.31$ & $17.033 \pm 5.12$ \\
\hline 5 & 17.88 & 18.58 & 10.37 & 13.02 & 13.94 & 15.12 & 16.51 & 16.82 & $14.68 \pm 3.30$ & $15.89 \pm 2.38$ \\
\hline 6 & 15.30 & 17.83 & 9.20 & 9.38 & 13.50 & 15.81 & 13.1 & 12.31 & $12.78 \pm 2.57$ & $13.83 \pm 3.74$ \\
\hline Mean \pm SD $(\mathrm{mm})$ & \multicolumn{2}{|c|}{$19.29 \pm 3.47$} & \multicolumn{2}{|c|}{$10.52 \pm 1.79$} & \multicolumn{2}{|c|}{$15.73 \pm 2.60$} & \multicolumn{2}{|c|}{$15.87 \pm 3.34$} & & \\
\hline Coefficient of Variation (\%) & \multicolumn{2}{|c|}{18.0} & \multicolumn{2}{|c|}{17.0} & \multicolumn{2}{|c|}{16.5} & \multicolumn{2}{|c|}{21.0} & & \\
\hline
\end{tabular}




\section{Table 2 (on next page)}

The cross-sectional areas of cranial cruciate ligaments (CCL) for CCLs in paired canine stifle joints ( $n=6$ pairs). 


\begin{tabular}{|c|c|c|}
\hline No. & \multicolumn{2}{|c|}{ Cross-sectional area $\mathbf{( m m}^{2}$ ) } \\
\hline $\mathbf{1}$ & Right CCL & Left CCL \\
\hline $\mathbf{2}$ & 12.58 & 14.99 \\
\hline $\mathbf{3}$ & 14.39 & 14.41 \\
\hline $\mathbf{4}$ & 15.48 & 13.98 \\
\hline $\mathbf{5}$ & 12.91 & 15.69 \\
\hline $\mathbf{6}$ & 14.93 & 23.62 \\
\hline Mean \pm SD (mm $\left.{ }^{2}\right)$ & $16.10 \pm 5.10$ \\
\hline Coefficient of Variation (\%) & \multicolumn{2}{|c|}{31.7} \\
\hline
\end{tabular}


Figure 1

A typical stress strain curve of a knee joint ligament loaded to failure, illustrating the three major regions of the curve.

$\mathrm{R} 1$ : toe region where the ligament fibres tighten, and crimp is removed. R2: elastic region. R3: plastic region. The graph is based on previous literature including Aims 2004 and Wingfield et al. 2000.

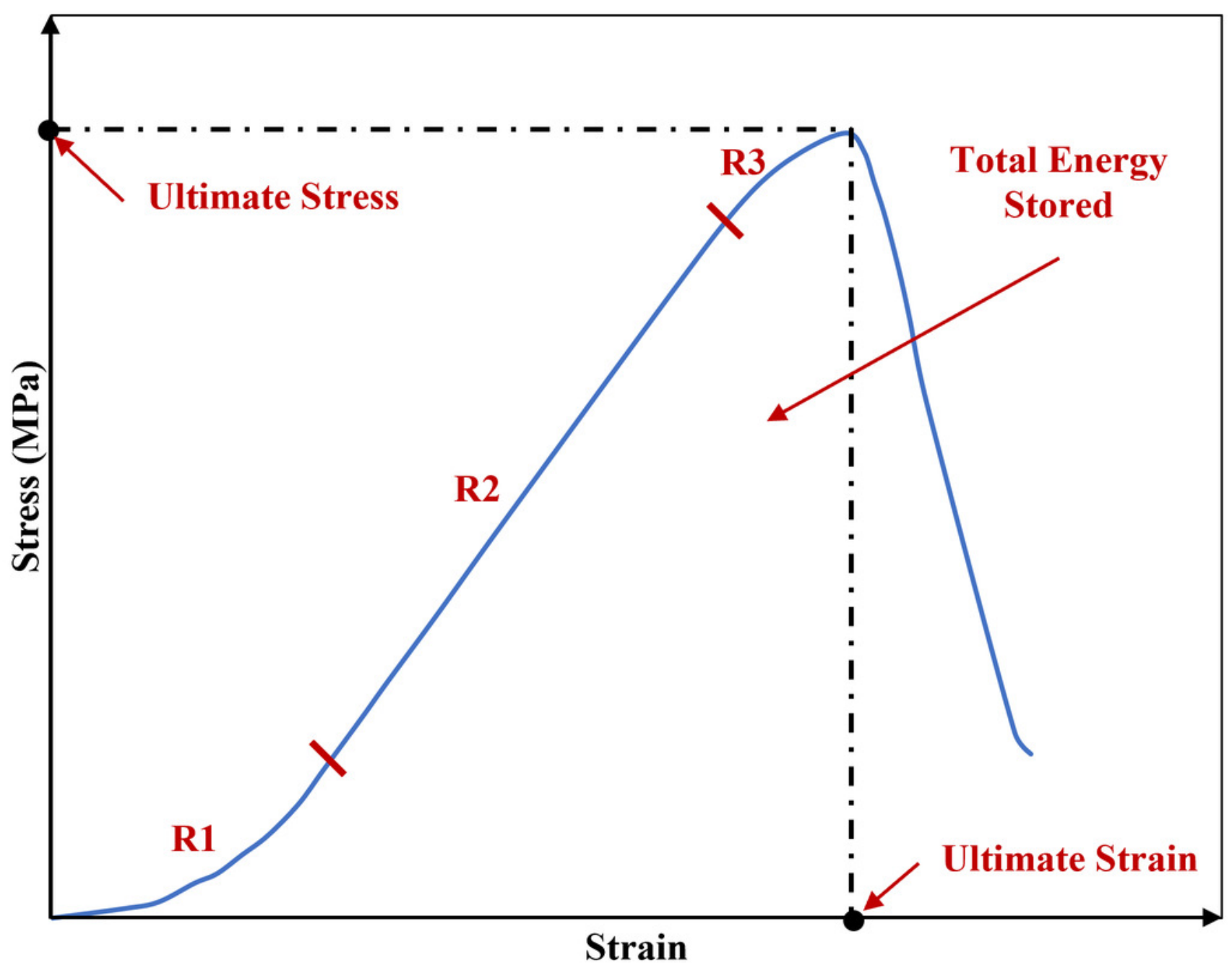


Figure 2

The extracted cranial cruciate ligaments (CCL) consisted of approximately $10 \mathrm{~mm}$ of the femoral and tibial bones forming femur-CCL-tibia complex. 


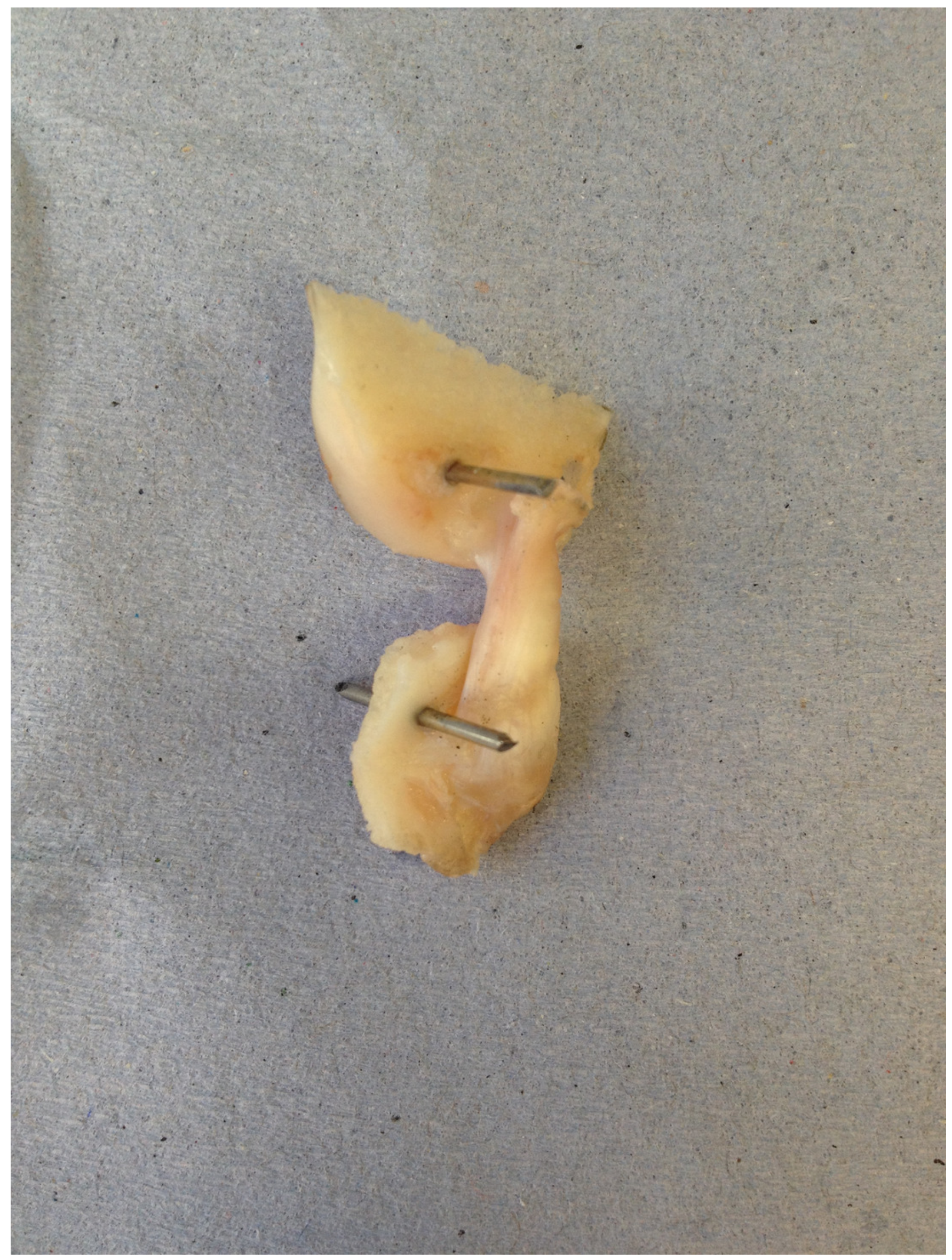




\section{Figure 3}

The uniaxial experimental test setup.

(A) Showing the design of the custom-made clamps that was used for (B) manufacturing the parts of the experimental testing rig. The custom-built clamps included a (i) cylindrical

Perspex tank, (ii) lower and (iii) top grips, (iv) duck-tail sandwich clamps and (v) screws. (C) An example of a cranial cruciate ligament (CCL) clamped and placed in the Perspex tank. 


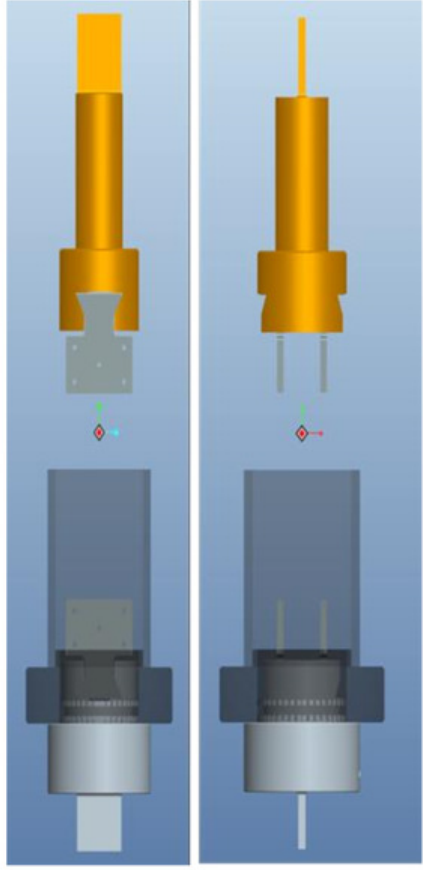

(A)

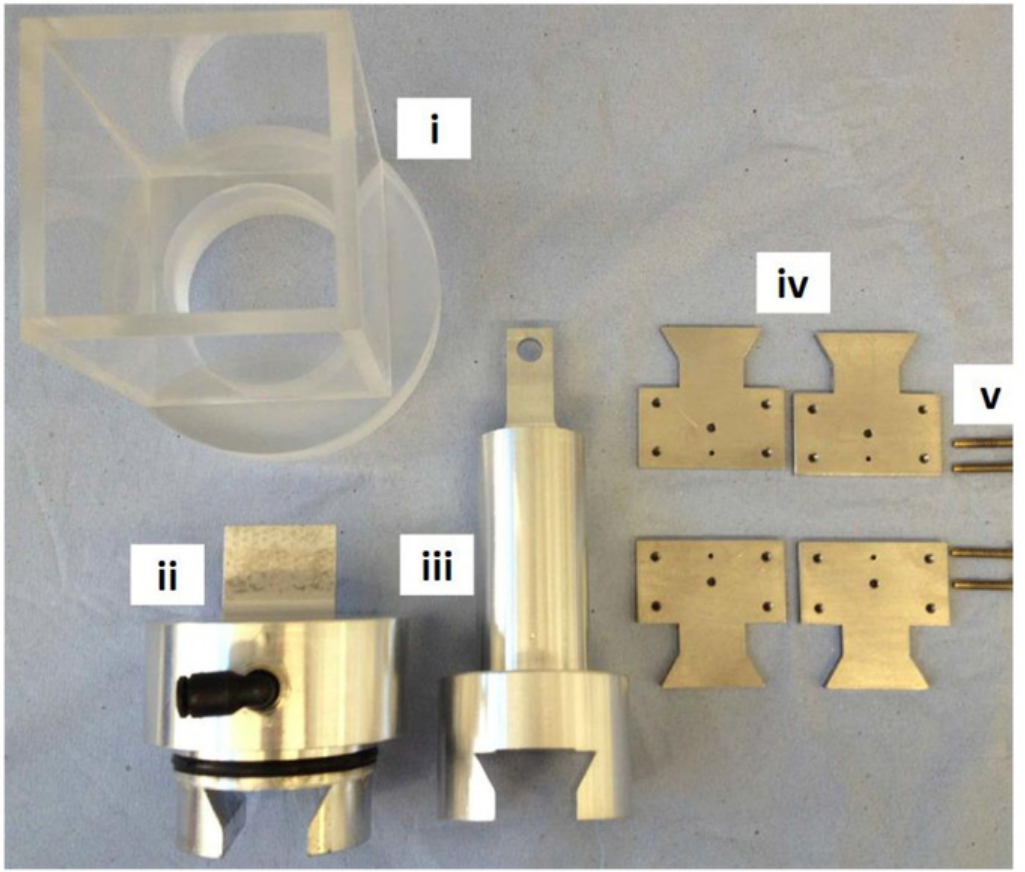

(B)

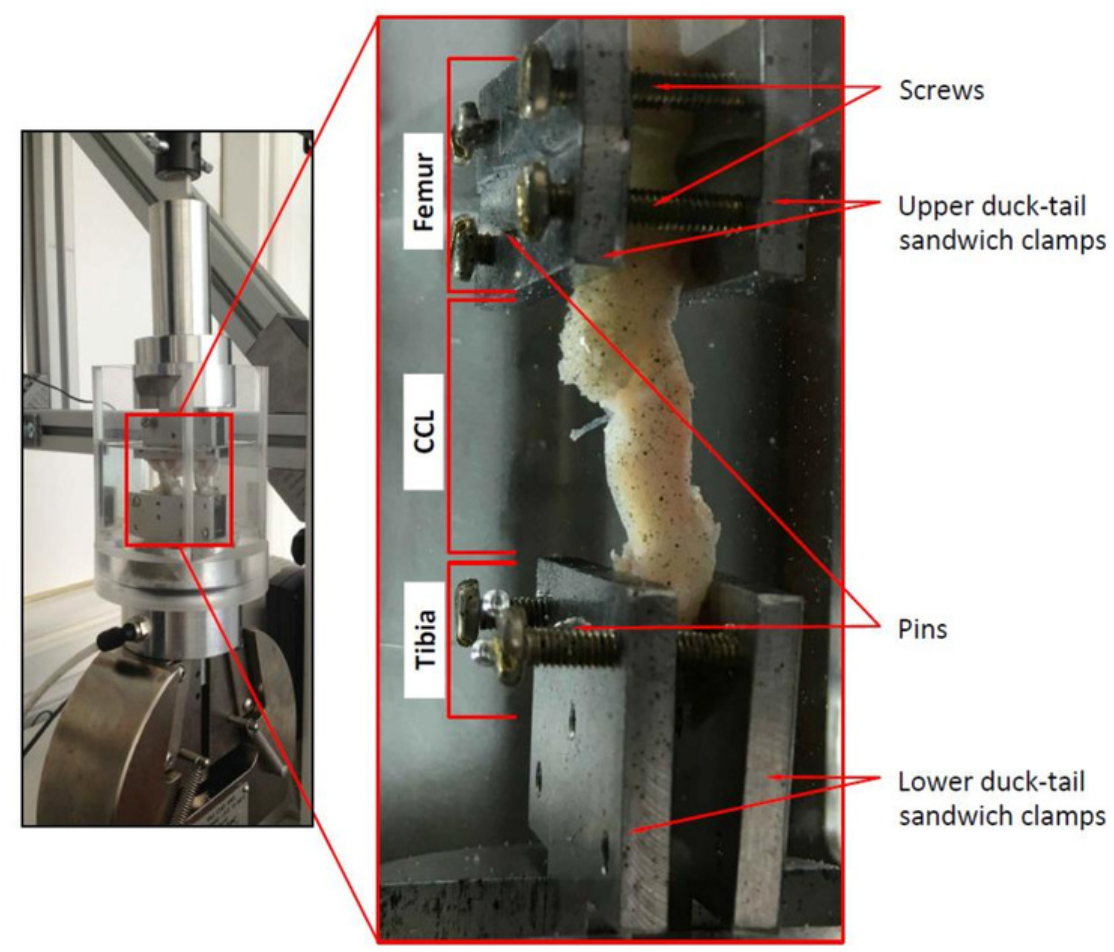

(C) 
Figure 4

The tensile characteristics of a canine cranial cruciate ligament (CCL) following ascending (Asc) and descending (Desc) protocols at $0.1,1$ and $10 \% /$ min strain rates.

(A) A typical cyclic loading and unloading stress-strain curves and (B) tangent modulus-stress behaviour of the loading curves of a CCL at varying strain rates.

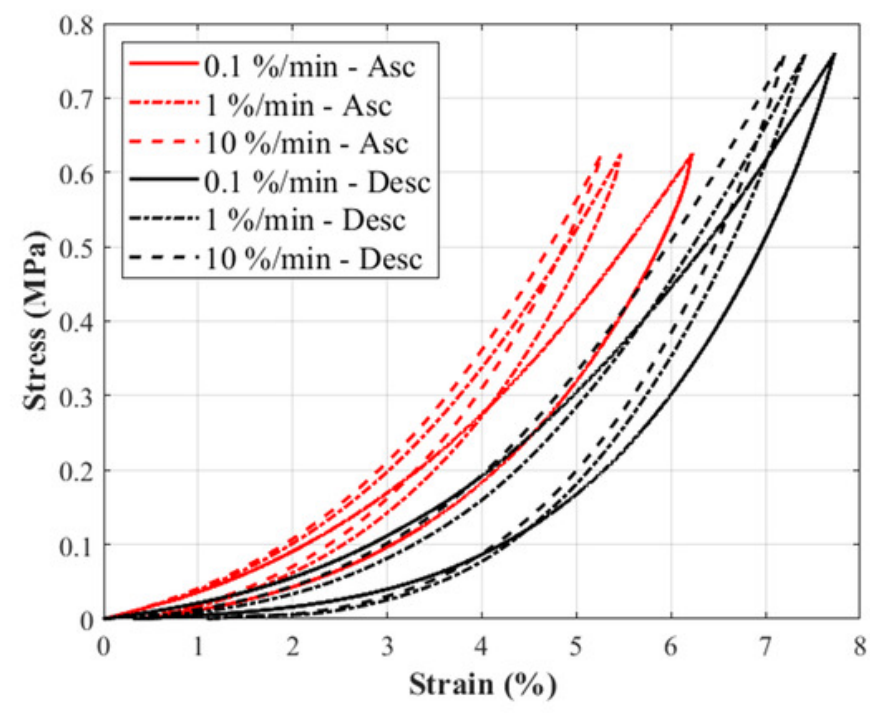

(A)

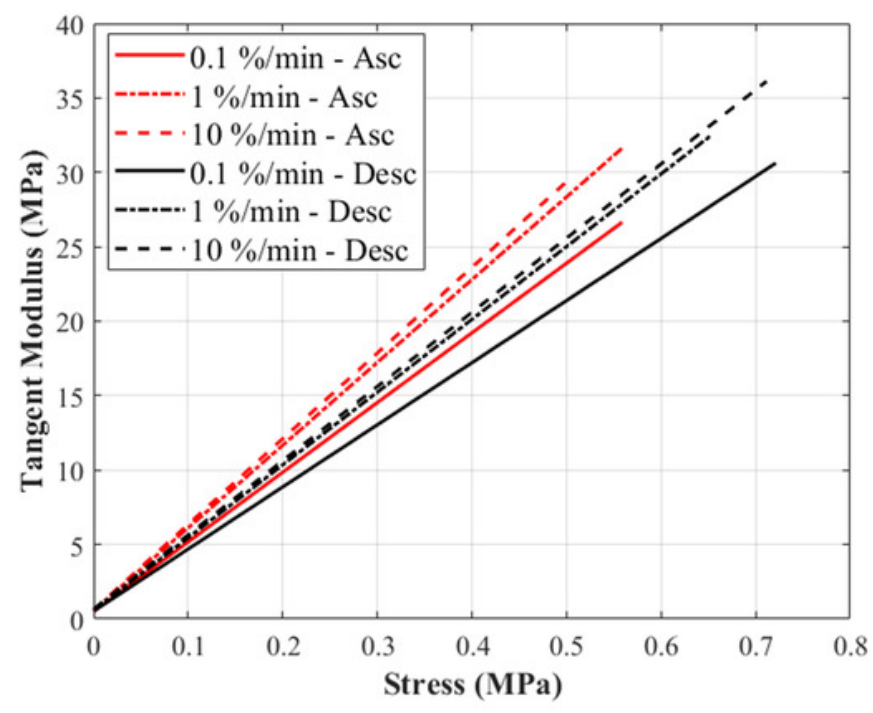

(B) 


\section{Figure 5}

Tensile behaviour of the canine cranial cruciate ligaments was investigated following ascending (red line) and descending (black line) protocols at varying strain rates.

The box plots show specimen variation and stress at 1, 3 and $5 \%$ strain during loading at (A) $0.1 \% / \mathrm{min}$, (B) $1 \% / \mathrm{min}$ and (C) $10 \% / \mathrm{min}$ strain rates, and tangent modulus at $0.1,0.3$ and $0.5 \mathrm{MPa}$ stress during loading at (D) $0.1 \% / \mathrm{min}$, (E) $1 \% / \mathrm{min}$ and (F) $10 \% / \mathrm{min}$ strain rates. The outliers are indicated with a red plus sign. 


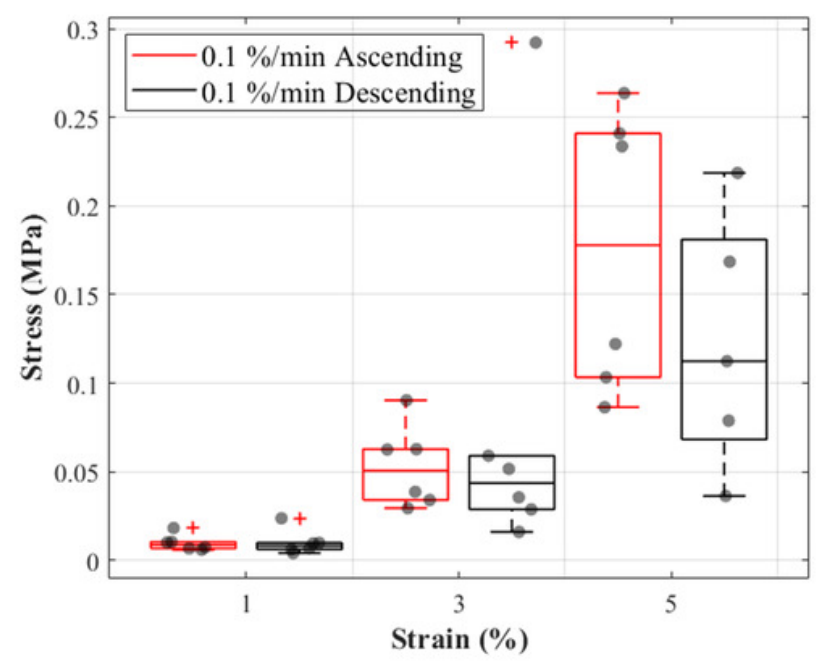

(A)

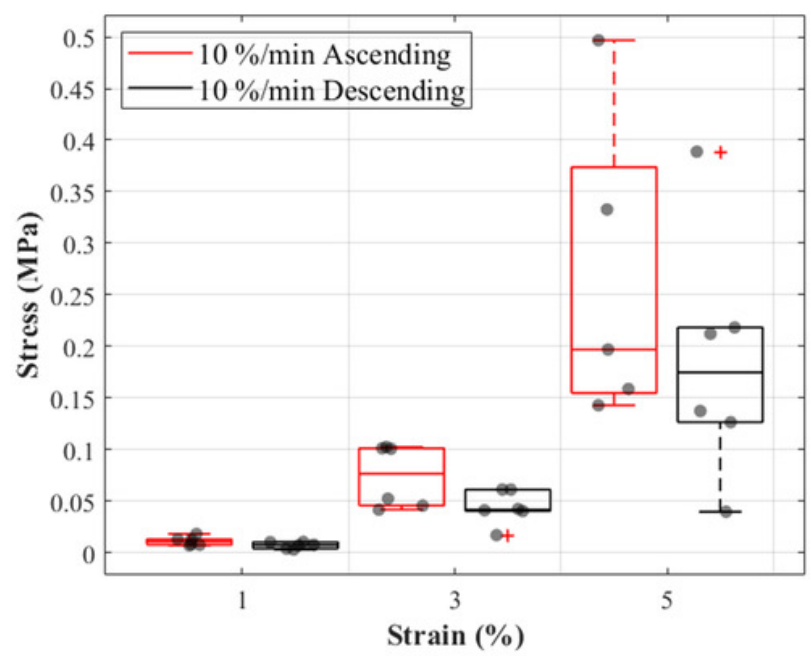

(C)

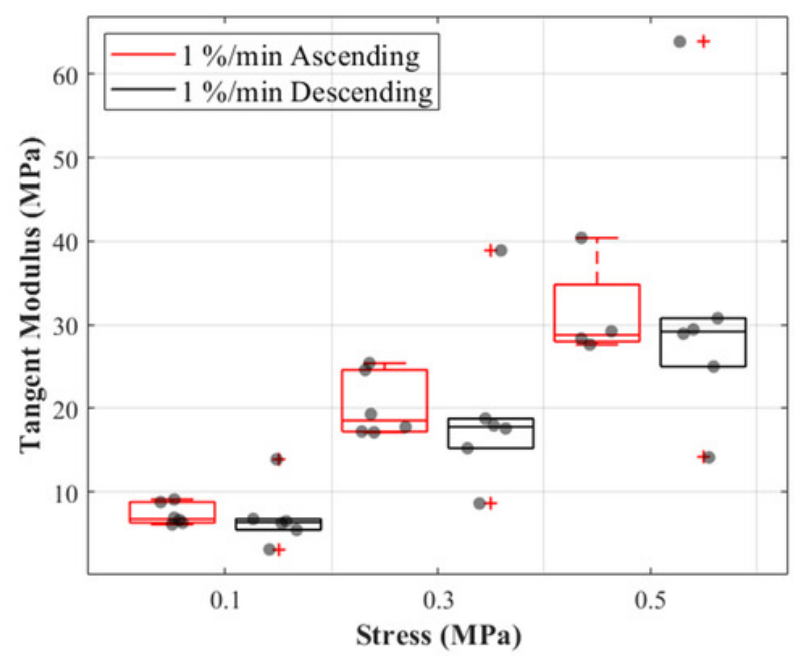

(E)

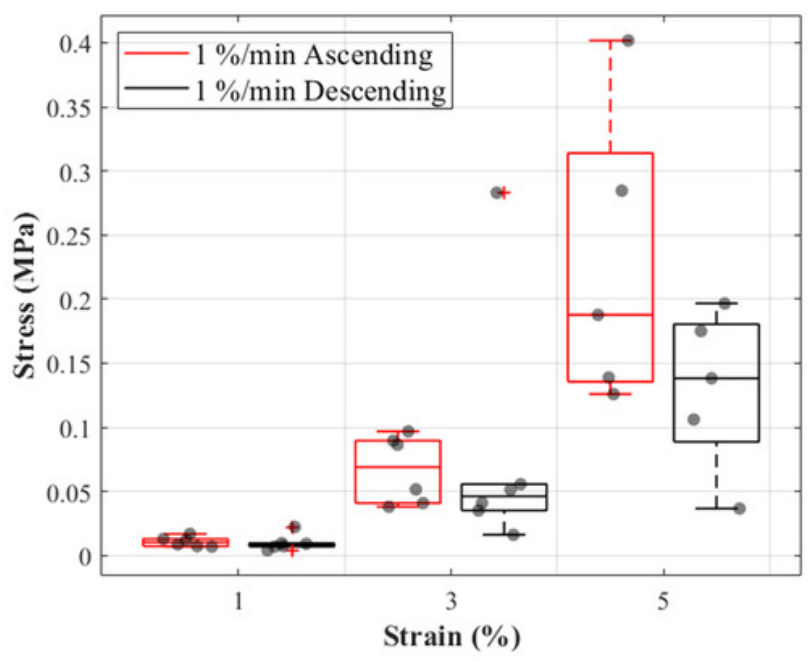

(B)

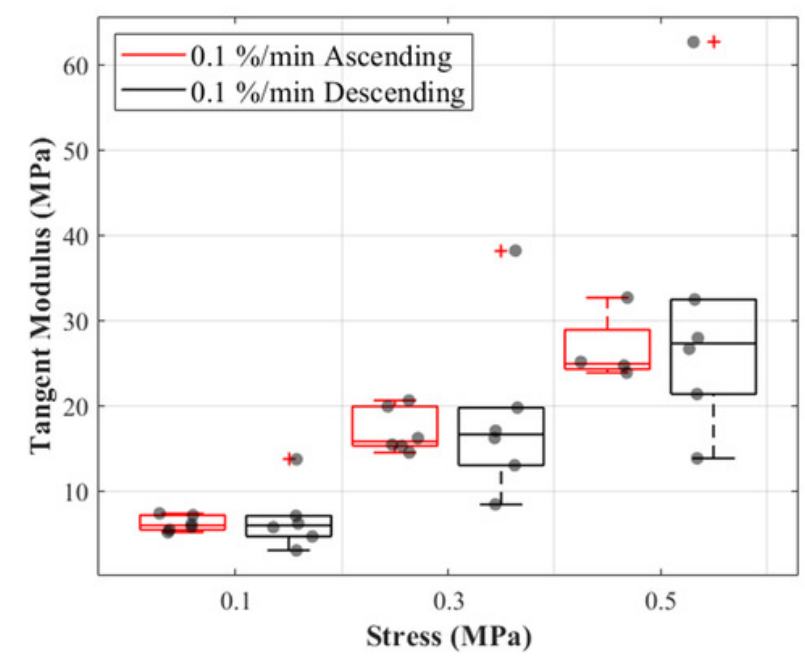

(D)

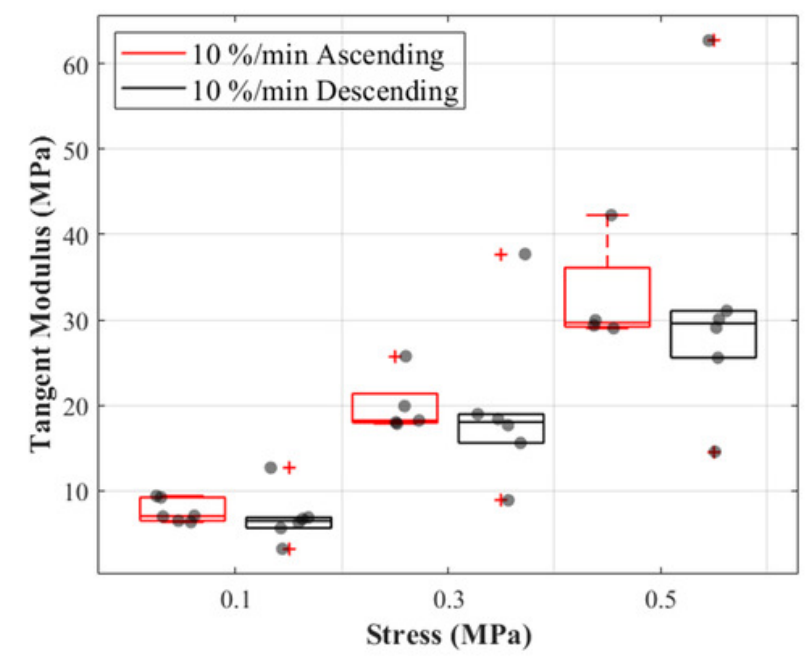

(F) 


\section{Figure 6}

Dissipated energy of the canine cranial cruciate ligaments (CCL) during cyclic loading at varying strain rates.

(A) The decrease in mean dissipated energy with increasing cycles of loads. The first ten cycles represent dissipated energy during the precondition stage of the CCLs. From cycles 11 to 19 dissipated energy values are associated with CCLs during tensile tests at three different strain rates $(0.1,1$ and $10 \% / m i n$ with each test repeated three times). The ascending testing protocol (red line) resulted in a slightly lower dissipated energy compared to the descending (black line) testing protocol. (B) Variations in dissipated energy within the specimens. The median values (indicated by the horizontal line inside the boxes) show a decrease in dissipated energy (hysteresis) with increasing strain rates. However, this observation was only statistically significant (blue line and ${ }^{*}$ ) between tensile tests at strain rates of 0.1 and 1 $\% / \mathrm{min}$, and 0.1 and $10 \% / \mathrm{min}$.

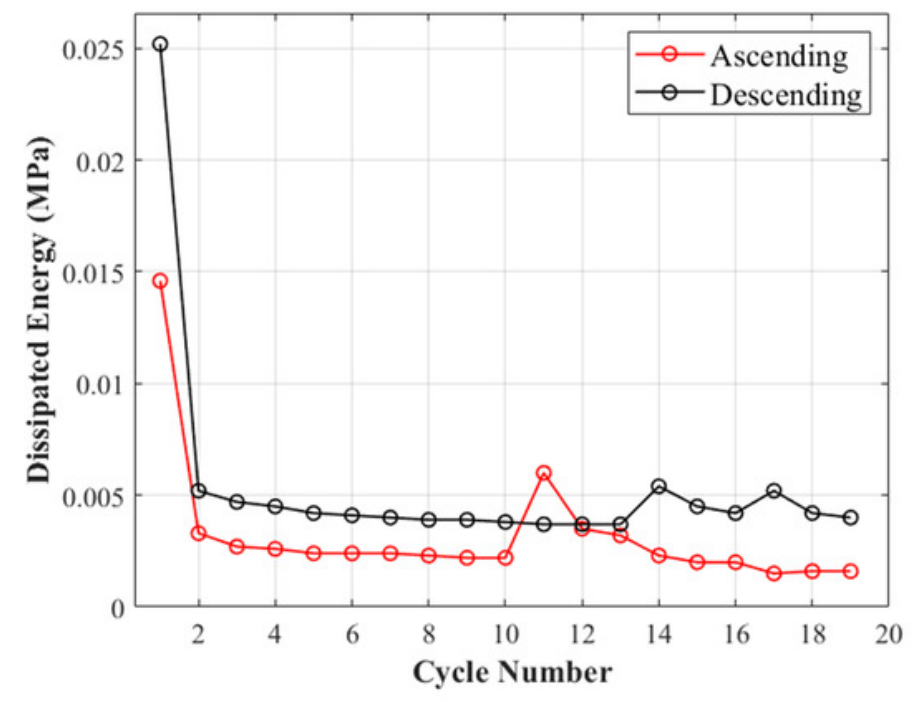

(A)

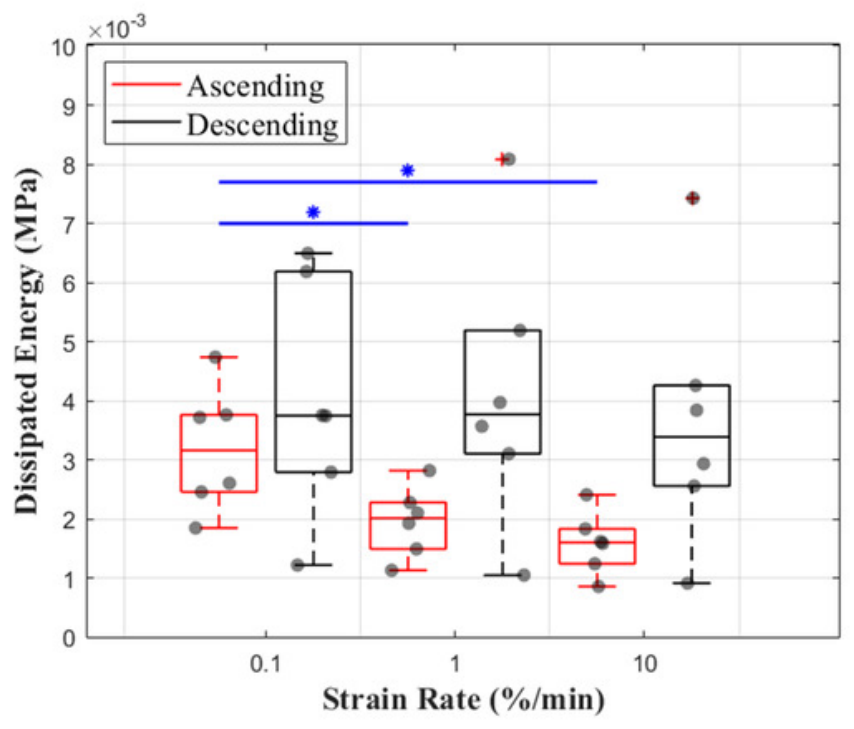

(B) 


\section{Figure 7}

Recovery of the canine cranial cruciate ligaments (CCL) during cyclic loading at varying strain rates.

(A) The average recovered length of the CCLs at different cycles. During the preconditioning cycles (the first ten cycles) recovered lengths of the ligaments are similar. Cycles associated with mechanical tests (cycles 11 to 19) for both testing protocols (ascending in red and descending in black) showed an increase in recovery with increasing strain rates. (B) Variations in length recovery within the CCLs. The box plots show an increase in recovery with increasing strain rates. This characteristic was statistically significant between tensile tests at strain rates of 0.1 and $1 \% / \mathrm{min}$, and 0.1 and $10 \% / \mathrm{min}$ as indicated by the blue $*$ and line.

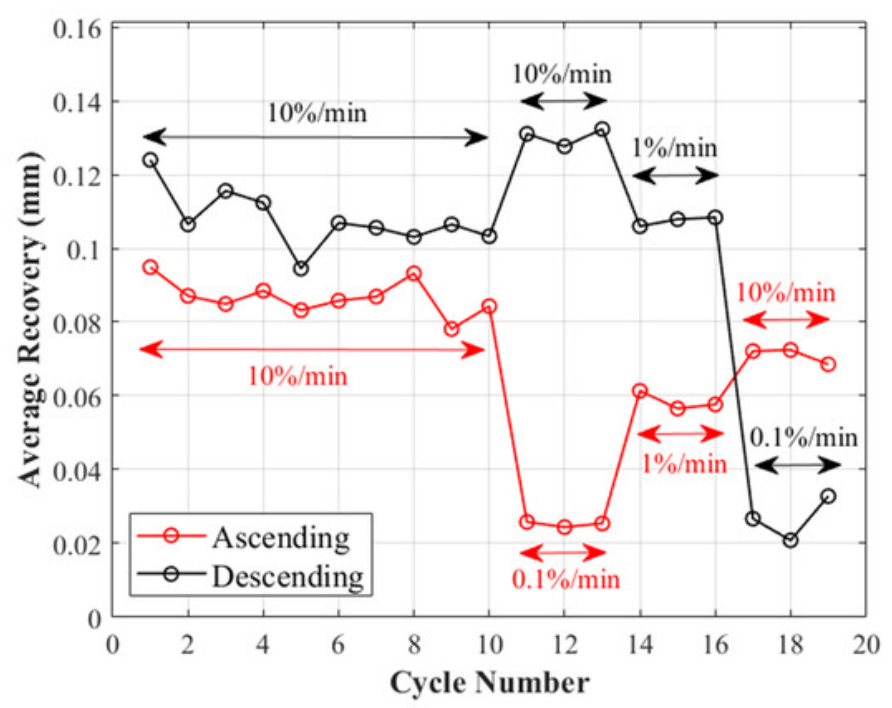

(A)

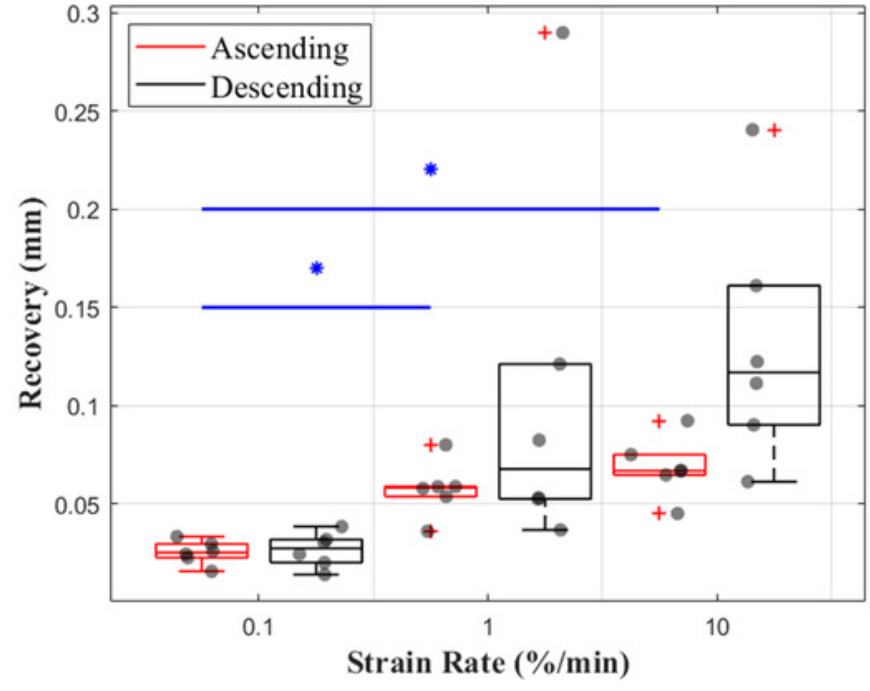

(B) 